\title{
Edorexia y deporte. Una concepción acerca de la obesidad y la adicción a la comida
}

\section{Edorexia and Sport. A Conception of the Obesity and Craving Food}

\author{
José Luis López Morales y Enrique Javier Garcés de los Fayos Ruiz
}

Universidad de Murcia, España

\begin{abstract}
Resumen: Este estudio tiene como objetivo analizar la dieta hipocalórica y el deporte en el tratamiento de la obesidad y sus componentes como la adicción a la comida. El deporte es una de las herramientas más utilizadas para tratar enfermedades como la obesidad. Sin embargo, los últimos avances en la Psicología concluyen como la obesidad oculta un problema de control de impulsos relacionado con el apetito. En este estudio se deduce como la dieta hipocalórica y el deporte tiene efectos secundarios y no tratan el problema oculto de la obesidad. Además, los deportistas, entre otros colectivos, pueden emplear estas herramientas para impedir que se manifieste la obesidad y sus componentes como la adicción a la comida, reforzando las conductas y los pensamientos de riesgo y provocando un empeoramiento del problema. Palabras clave: edorexia, obesidad, deporte, dieta, tratamiento, adicción a la comida.
\end{abstract}

Abstract: This study aims to analyze how low-calorie diets and sports operate on the treatment of obesity, as well as its components such as craving food. Sport is one of the most common resources used in order to treat diseases such as obesity. Nevertheless, recent advances in Psychology conclude the existence of an impulse control disorder related to appetite behind obesity. This study deduces that low-calorie diets and sports have secondary effects and do not address the underlying problem of obesity. In addition, athletes - among other collectivities - can use these resources to avoid the manifestation of obesity and so its components such as food addiction, reinforcing risk behaviors and thoughts and worsening the problem. Key Words: edorexia, obesity, sport, diet, treat, craving food.

\section{Introducción}

La alimentación y el deporte se encuentran estrechamente relacionados. La practica deportiva implica unas mayores demandas de energía y nutrientes, por ello el deportista debe consumir más cantidad de alimentos que la población sedentaria (Arasa, 2005); esta relación es bidireccional, es decir, por un lado se produce una demanda energética por el esfuerzo físico que exige la práctica del deporte, y por otro el rendimiento deportivo es el resultado de muchos factores, entre las que destaca la alimentación, una alimentación inadecuada dificulta el rendimiento máximo de un deportista. Además, se emplea el deporte como una herramienta para facilitar el control del gasto energético y por ello es uno de los principales módulos en los tratamientos de enfermedades como la obesidad (Consenso FESNAD-SEEDO, 2011; Summerbell et al., 2012). Se entiende la obesidad como un desequilibrio energético entre el aporte calórico y el gasto energético, por lo tanto, el tratamiento principal consiste en la disminución del primer factor y el aumento del segundo, mediante una dieta hipocalórica y la realización de ejercicio físico, respectivamente. Sin embargo, existen otros factores que también intervienen y no se presenta atención en los estudios y programas de intervención sobre esta patología como por ejemplo, estrés, ansiedad, adicción a la comida, depresión, trastornos del sueño o el mecanismo homeostático energético, entre otros

José Luis López Morales (psicólogo y becario de investigación). Universidad de Murcia, Facultad de Psicología, Campus de Espinardo, 30100, Murcia (España). Email: joseluis.lopez2@um.es
(Corwin y Grigson, 2009; Elder et al., 2011; Timko y Perone, 2004), en los cuales se relaciona la relación de la motivación de comer con la depresión, estrés y con la adicción a la comida; aunque no siempre se relacionada con un incremento del indicé de masa corporal, indicando un exceso de peso, es decir, estos indicadores se relaciona con un incremento apetito, diferenciando con la ingesta y, además, se establecen los diferentes componentes que integran la obesidad.

\section{Una aproximación conceptual a la obesidad y sus componentes: Edorexia}

Como se presentó en el XIII Congreso Nacional y I Foro Mediterráneo de Psicología de la Actividad Física y del Deporte en Murcia, existen una amplia variedad de investigaciones psicológicas relacionadas con la obesidad, durante este articulo se citaran las más destacas. La mayor parte de ellas tienen como finalidad estudiar la obesidad como una adicción a la comida, hallándose semejanzas a nivel estructural con otras adicciones ya que comparten los mismos mecanismos de acción (Avena, 2008; Rolls y McCabe, 2007; Wise, 2006), por lo que algunos expertos de la Psicología consideran apropiado asociar la comida con una droga (Muele, 2011; Volkow et al. 2008). Sin embargo, el Código Alimentario Español (Decreto 2484/1967) define los alimentos como se perciben generalmente, sin considerarlos una sustancia tóxica, "todas las sustancias o productos de cualquier naturaleza, sólidos o líquidos, naturales o transformados, que por sus caracte- 
rísticas, aplicaciones, componentes, preparación y estado de conservación, sean susceptibles de ser habitual o idóneamente utilizados a alguno de los fines siguientes: a) para la normal nutrición humana o como fruitivos, y b) como productos dietéticos, en casos especiales de alimentación humana."; por todo ello, resumir la adicción a la comida y considerar que la comida es una droga es inapropiado, se requiere de una contextualización: en primer lugar, los alimentos son todas aquellas sustancias que necesitamos para nuestra supervivencia; en segundo lugar, nuestros antepasados requerían de un gran esfuerzo para conseguir los alimentos por lo que es lógico que el organismo haya ingeniado estrategias para promocionar esas conductas y conseguir los alimentos; las cuales, forman actualmente parte de nuestra herencia genética. Por ejemplo, se ha comprobado como se producen alteraciones neuronales con el consumo de azucares pero no se produce lo mismo con las grasas (Avena, 2009); ya que nuestro sistema nervioso requiere de una constante suministro de hidratos de carbono, convirtiéndose en una estrategia vital para nuestra supervivencia en épocas de ayuno o escasez de comida. No obstante, Pelchat (2009) asegura que no se puede considerar la comida como una sustancia adictiva, ya que no se produce en todos los casos las consecuencias negativas características de una sustancia de abuso. Por consiguiente, la adicción a la comida formaría parte de las estrategias del organismo para su supervivencia vital en un ambiente determinado y sin consecuencias negativas; aunque puede convertirse en disfuncional como sucede con la ansiedad, entre otras.

Por otra parte, y siguiendo el planteamiento realizado hasta ahora, López Morales y Garcés de los Fayos (en prensa) muestran un modelo integrador englobando los avances psicológicos con los conocimientos de la nutrición. En esta perspectiva, destaca la atención hacía el apetito como el principal problema y el balance de los factores que intervienen en la obesidad (aporte calórico, gasto energético, mecanismo hemostático energético y vulnerabilidad genética); en resumen, el comer por apetito sin control sería el desencadenante del exceso de peso. Demos, Heatherton y Kelley (2012) concluyen que se produce un aumento de la actividad del núcleo accumbens en presencia de imágenes de alimentos y correlaciona positivamente con un aumento del apetito, también concluyen la existencia de la falta de control en los individuos que comen en exceso. Teniendo en cuenta estos datos, se puede concluir como la obesidad y sus componentes (principalmente la adicción a la comida) son el resultado de un apetito descontrolado producido por un déficit de control, semejante a un problema de control de impulsos en vez de una dependencia a una sustancia; a partir de aquí lo denominaremos edorexia por tratarse de un término que se compone de dos palabras latinas (edo y orexis, que significan comer y apetito, respectivamente) y enfatiza las conductas problemas asociadas a la obesidad como son el apetito producido por diversos modos: la adicción a la comida, el estrés o por episodios ansiosos/depresivos.

Siguiendo esta perspectiva, no se pretende restar importancia a la obesidad, sino redefinirla de una manera más completa. Para que un individuo consiga engordar (de manera exógena) requiere el cumplimiento de unas condiciones: el aporte calórico sea superior al gasto energético, disponer una vulnerabilidad genética propicia en un ambiente idóneo (Friedman, 2003) y tener alterado el mecanismo de homeostasis energética o ignorar las señales de saciedad para satisfacer otras necesidades internas (apetito). Por lo que, los individuos que desarrollan edorexia no significan que necesariamente terminen mostrando un exceso de peso, ya que además del aporte calórico hay que prestar atención a los demás factores (López Morales y Garcés de los Fayos, en prensa). No obstante, si pueden desarrollar el miedo a engordar típico de la obesidad y de otros trastornos de la conducta alimentaria. En futuras investigaciones se tiene que confirmar la presencia de este síntoma en la población obesa y no obesa, como el colectivo de deportistas. Una de las características principales de éstos es su alto gasto energético, permitiéndose ocultar una posible edorexia manifestada por ejemplo en una adicción a la comida. Muele (2011) comenta los datos de un estudio en el cual se encuentra que el $16.3 \%$ de la muestra fue diagnostica como adicta a la comida sin presentar un exceso de peso. Sin embargo, no existen estudios empíricos que describan la relación de la adicción a la comida con el colectivo deportivo.

Por otro lado, ya se ha mencionado que las recomendaciones y el tratamiento de primer orden son la dieta hipocalórica y el ejercicio físico; desde un análisis psicólogo más profundo Massey y Hill (2012) en su estudio concluyen que se produce un incremento del apetito con la realización de una dieta. Además, al analizar las dietas se identifican castigos positivos al obligar consumir ciertos alimentos percibidos como desagradables para el sujeto (en algunos casos son verduras o pescados); un castigo negativo al prohibir algunos alimentos agradables (en este caso, suelen ser dulces) y un reforzamiento negativo de la conducta de riesgo al producir una situación de escape de la obesidad. En definitiva, se refuerza todas aquellas conductas (tanto los hábitos alimentarios como las creencias y los pensamientos) que han desencadenado la obesidad y generan un incremento en el apetito; por lo que en el mejor de los casos se mantiene la edorexia (manteniéndose también el problema principal) o incluso desencadenando un mayor apetito, lo que influirá en la manifestación posterior de una obesidad. Del mismo modo, la realización del deporte puede entenderse como un escape de la obesidad, controlando uno de los principales factores para manifestar un exceso de peso.

\section{Conclusión}

En este estudio se focaliza la atención en los aspectos psico- 
lógicos presentes en la obesidad, sus componentes (ansiedad, depresión, estrés o adicción a la comida) que promueven el exceso de peso y se realiza una revisión y un análisis de los tratamientos de primer orden. Se parte del modelo integrador comprensivo de la obesidad (López Morales y Garcés de los Fayos, en prensa) donde se establecen cuatro factores que intervienen en la obesidad: aporte calórico, gasto energético, mecanismo hemostático energético y vulnerabilidad genética.

En este análisis, se haya el apetito y el déficit de control como los verdaderos problemas presentes en la sociedad y que intervienen en las diferentes facetas de la vida de los individuos (Massey y Hill, 2012; Villaseñor Bayardo, Onnteveros Esqueda y Cárdenas Cibrián, 2006); el exceso de peso se considera como una manifestación de estas características, que a su vez, pueden estar presentes también en otros colectivos como los deportivas. Futuras investigaciones tienen que confirmar estas hipótesis.

La dieta hipocalórica considerada como el principal tratamiento de la obesidad (consenso FESNAD-SEEDO, 2011), corresponde a un tratamiento inadecuado para actuar contra la edorexia presente en los individuos con obesidad y que co- rresponde a los factores que originan y mantienen la obesidad. Por otro lado, el deporte sigue siendo uno de las principales herramientas para controlar el gasto energético; aunque puede utilizarse también como un escape de la obesidad, provocando un reforzamiento negativo y manteniendo el problema. Del mismo modo, la dieta hipocalórica y el deporte puede ser empleado con los mismos objetivos en individuos con edorexia sin demostrar obesidad, por ese motivo, es necesario que futuras investigaciones presten atención a estos síntomas para prevenir este problema. Por último, futuras investigaciones deben presentar unas estrategias más adecuadas para tratar la edorexia en la población obesa y en otros individuos, sin utilizar aprendizaje por castigos o reforzamientos negativos, se debe establecer una estrategia basada en los reforzamientos positivos y tratando los diferentes aspectos que engloba este problema: aspectos nutricionales, estilos de vida (sueño y deporte) y psicológicos (autoestima, estrés, ansiedad, percepción interoceptiva y control de estimulo) y como lo indican las publicaciones de Elder et al. (2011), Michaelides (2011), Timko y Perone (2004) o Summerbell et al. (2012), entre otros.

\section{Referencias bibliográficas}

Arasa Gil, M. (2005). Manual de nutrición deportiva. Barcelona: Editorial Paidotribo.

Avena, N.M, Rada, P. y Hoebel, B.G. (2009). Sugar and fat bingeing have notable differences in addictive-like behavior. The Journal of Nutrition, 139(3), 623-628.

Avena, N.M., Rada, P. y Hoebel,B.G. (2008). Evidence for sugar addiction: Behavioral and neurochemical effects of intermittent, excessive sugar intake. Neuroscience Biobehavior, 32, 20-39.

Corwin, R.L. y Grigson, P.S. (2009). Symposium overview. Food addiction: Fact of fiction?. The Journal of Nutrition, 139, 1S-3S.

Demos, K.E., Heatherton, T.F. y Kelley, W.M. (2012). Individual differences in nucleus accumbens activity to food and sexual images predict weight gain and sexual behavior. The Journal of Neuroscience, 32(16):5549 -5552.

Elder, C.R., Guillion, K.L., Funk, K.L., BeBar, L.L., Lindberg, N.M. y Stevens, V-J. (2011). Impact of sleep, screen time, depression and stress on weight change in the intensive weight loss phase of the life study. International Journal of Obesity, 36(1), 86-92.

FESNAD-SEEDO (2006). Recomendaciones nutricionales basadas en la evidencia para la prevención y el tratamiento del sobrepeso y la obesidad en adultos (Consenso FESNAD-SEEDO). Revista Española de Obesidad, 10(1), 1-80.

Friedman, J.M. (2003). A war on obesity, not the obese. Science, 5608(299) ,856-858.

López Morales, J.L., Garcés de los Fayos, E. (En revisión). Hacia una integración comprensiva de la obesidad desde una perspectiva multidisciplinar. Nutrición Hospitalaria.
Meule, A. (2011). How prevalent is "food addiction". Frontiers in Psychiatry, 2, 61-64.

Michaelides, M. (2011). Functional neuroimaging in obesity. Psychiatric Annals, 41(10), 496-500.

Pelchat, M. (2009). Food addiction in humans. The Journal of Nutrition, 139 (3), 620-622.

Peters, A. (2012). Does sugar addiction really cause obesity?. Frointers in Neuroenergetics, 3, 1-2.

Rolls, E.T. y McCabe, C. (2007). Enhanced affective brain representations of chocolate in cravers vs. non-cravers. European Journal of Neuroscience, 26, 1067-1076.

Summerbell, C.D., Moore, H. J., Vogele, C., Kreichauf, S., Wildgruber, A., Manios, Y., Douthwaite, W., Nixon, C. A. y Gibson, E. L. (2012). Evidence-based recommendations for the development of obesity prevention programs targeted at preschool children. Obesity Reviews, 13, 129-132.

Timko, C.A. y Perone, J. (2004). Rigid and flexible control of eating behavior in a college population. Eating Behaviors, 6, 119-125.

Villaseñor Bayardo, S.J., Onnteveros Esqueda, C. y Cárdenas Cibrián, K.V. (2006). Salud mental y obesidad. Investigación en salud, 7(2), 86-90.

Volkow,N.D., Wang, G.J., Fowler,J.S. y Telang,F.(2008). Overlapping neuronal circuits in addiction and obesity: evidence of systems pathology. Philosophical Transactions of the Royal Society Biological Sciences, 363(1507), 3191-3200.

Wise, R.A. (2006). Role of brain dopamine in food reward and reinforcement. Philosophical Transactions, 361, 1149-1158. 